\title{
The effects of pain on quadriceps strength, joint proprioception and dynamic balance among women aged 65 to 75 years with knee osteoarthritis
}

\author{
Dokyung Kim', Geon Park', Liang-Tseng Kuo ${ }^{2,3^{*}+}$ (D) and Wonhah Park ${ }^{1+}$
}

\begin{abstract}
Background: Patients with knee osteoarthritis $(\mathrm{OA})$ were reported to have quadriceps weakness, and impaired proprioception, both related to pain and swelling. It is unclear whether pain alone a causal factor to above findings over the knee joint. The purpose of this study was to assess the effects of knee pain alone on the quadriceps strength, proprioception and dynamic balance in subjects with bilateral knee OA without joint swelling.

Methods: Fourty females with mean age of 68.3 years were involved in this cross-sectional study. The inclusion criteria were bilateral knee OA without joint swelling, with a visual analogue pain scale difference $(>1)$ between each knee. Patients all underwent assessment of the isokinetic strength of knee muscles, knee proprioceptive acuity, and dynamic balance.

Results: Patients' more painful knee had weaker isokinetic quadriceps strength than less painful knee at both $60 \% \mathrm{~s}$ and $180 \%$ ( $(p=0.01, p=0.01$, respectively). There were no differences in proprioceptive acuity between both knees in all three knee positions. Meanwhile, there was a significant difference in the dynamic balance index measurement between both knees (more painful versus less painful: $3.88 \pm 1.15$ vs. $3.30 \pm 1.00, p=0.01$ ). Quadriceps strength was associated with dynamic balance stability $(60 \% \mathrm{~s}, r=-0.578, p<0.01 ; 180 \% \mathrm{~s}, r=-0.439, p<0.01)$.

Conclusions: For patients with knee OA, the more painful knee was associated with weaker quadriceps and poor balance ability. To improve lower limb function and balance stability of the older persons having knee OA, physicians should take the optimal pain management strategy.
\end{abstract}

Keywords: Knee osteoarthritis, Isokinetic knee strength, Pain, Proprioception, Dynamic balance

\section{Background}

Knee osteoarthritis $(\mathrm{OA})$ is a chronic disease that manifests as pain, stiffness, swelling and muscle weakness $[1,2]$, causes functional impairment due to lower limb muscle weakness, and is one of the most common diseases in the older persons [3-7]. A previous study reported that subjects with knee pain had a significantly higher incidence of quadriceps weakness after a 30-month period than subjects

\footnotetext{
* Correspondence: light71829@gmail.com

${ }^{\dagger}$ Liang-Tseng Kuo and Wonhah Park contributed equally to this work.

${ }^{2}$ Sports Medicine Center, Department of Orthopaedic Surgery, Chang Gung

Memorial Hospital, No. 6 West Sec, Chia-Pu Road, Putz city, Chiayi 613, Taiwan

${ }^{3}$ Chang Gung University of Science and Technology, Chiayi, Taiwan

Full list of author information is available at the end of the article
}

without pain and that patients with knee OA had a $76 \%$ reduction in eccentric quadriceps strength than healthy subjects [8]. Meanwhile, quadriceps muscle weakness may contribute to worsening of knee pain [9]. Also, quadriceps muscle strength is one of the intrinsic factors affecting the knee functions $[1,5]$.

Furthermore, patients with knee OA have impaired proprioception when compared to healthy people. Some studies described impaired proprioception as associated with the presence of pain [4], and knee pain was a significant predictor of decrease in balance. Also, the studies found that pain had effects, not only on muscle strength but also on proprioception $[10,11]$.

(c) The Author(s). 2018 Open Access This article is distributed under the terms of the Creative Commons Attribution 4.0 International License (http://creativecommons.org/licenses/by/4.0/), which permits unrestricted use, distribution, and reproduction in any medium, provided you give appropriate credit to the original author(s) and the source, provide a link to the Creative Commons license, and indicate if changes were made. The Creative Commons Public Domain Dedication waiver (http://creativecommons.org/publicdomain/zero/1.0/) applies to the data made available in this article, unless otherwise stated. 
In patients with knee OA, decreased muscle strength and proprioception are known to be affected by swelling as well as pain. Swelling is known to trigger the spinal inhibitory mechanism of quadriceps motor neurons, and to reduce muscle activity, thereby decreasing proprioception [12, 13]. However, there are varied opinions on the effects of knee pain alone on quadriceps muscle weakness, namely decreased proprioception and balance stability in patients with knee OA, without swelling $[14,15]$. It is unclear whether such functional impairments in patients with knee OA are due to swelling, or affected by the degree of pain, as related studies are scarce. There are also a few studies that designed to compare those results for each knee pain stage in OA and healthy subjects. Therefore, in this study, we aimed to investigate patients with knee OA without swelling that had different degrees of pain in each side of knee joints.

The purpose of this study was to investigate the effects of knee pain on quadriceps muscle strength, knee joint proprioception, and dynamic balance stability. We planned to compare outcomes between each knee in the same patient with knee OA. We also investigate the correlation between quadriceps muscle strength, knee joint proprioception, and dynamic balance stability.

\section{Methods}

\section{Subjects}

We conducted a cross-sectional study. Patients who visited our clinic due to knee pain were evaluated. Patients who met the following criteria were selected for the study:

1) Female patients, aged between 65 and 75 years, who had a diagnosis of knee OA according to the American College of Rheumatology criteria [16];

2) both knee joints with arthritic change grade 2 or higher in the Kellgren-Lawrence grading scale [17];

3) both knees have pain without swelling confirmed by ultrasonography, which performed by a senior radiologist who had performed ultrasonography for more than five years; and.

4) the difference of visual analogue scale (VAS) greater than one point between each knee joint.

The exclusion criteria were polyarthritis, the presence of rheumatoid arthritis or other systemic inflammatory arthropathies, any lower limb surgery within the last 12 months or a history of knee arthroplasty surgery, intraarticular corticosteroid injections into either knee in the previous month. Participants were also excluded if they had a neurologic or musculoskeletal condition that affected their balance or movement (i.e., Parkinson disease, multiple sclerosis).

All participants provided informed consent before testing, and the study was approved by the Sungkyunkwan
University School of Medicine Clinical Research Ethics Board (SMC 2017-05-084-001). An a priori power analysis was performed to determine the sample size using a two-sided hypothesis test at an alpha level of 0.05 and a power of 0.8 . The results of a previous study indicated that 25 knees would be required to detect a significant between-group joint proprioception difference of $>1^{\circ}$, the primary outcome measure [18]. The reporting of this study conforms to the STROBE statement [19].

\section{Measures}

Each subject had X-ray radiographs of both knees and completed pain evaluation as part of the study. Using the VAS scale, a score between 0 to 10 points of the combined pain in both knees was obtained, followed by separate scores for each knee, to identify the more and less painful. At the same time the quadriceps muscle strength, knee joints proprioception, and balance tests were performed after approximately $10 \mathrm{~min}$ of warm-up consisting of the stretches to hamstring, quadriceps, and calf muscles, and then a light level of stationary bike exercise. All observations and measurements were carried out by the same investigator.

\section{Knee muscle strength}

The strength and endurance of the knee extensor and flexor muscles were evaluated using an isokinetic dynamometer (CSMI Medical Solutions, MA, USA). The maximal strength of the quadriceps and hamstring was measured for each knee at $60 \%$ s (4 repetitions) and $180 \%$ s (20 repetitions), and mean power for each was calculated $[20,21]$. The maximum peak torque $(\mathrm{Nm})$ for each velocity was also recorded. This measure has excellent intra-rater reliability in patients with knee OA [22].

\section{Joint proprioception}

Knee joint proprioception was assessed using a Biodex System 3 (Biodex Medical, Shirley, NY, USA) and a knee joint repositioning task, previously used in those with knee OA to assess joint proprioception [23, 24]. Subjects were seated and blindfolded for the task. The leg was passively positioned and held for $10 \mathrm{~s}$ in 1 of 3 target positions $\left(15^{\circ}, 30^{\circ}\right.$, and $45^{\circ}$ of knee flexion). The knee was then returned to $90^{\circ}$ knee flexion, and the subject was asked to match the position their limb had been held. Two blocked trials at each of the three randomly presented target positions were completed, resulting in a total of six trials. Each trial was calculated with the absolute difference between actual leg position and target position degrees.

\section{Dynamic balance stability}

Dynamic Balance stability was measured with the Biodex Stability System (BSS, Biodex Medical Systems, Shirley, 
NY, USA). The reliability of this measurement was confirmed in a study by Wikstrom et al. [25]. The balance platform was set to tilt to all directions. All participants were trained one minute for adaptation to the machine, following which three practice trials, to reduce any learning effects, and three test evaluations were performed in each measurement session. The stability index represents the variance of platform displacement in degrees from level. The participant's ability to control the angle of tilt on the platform was measured by the system. The higher number of the index meant more motion, which indicates a problem with balance [26]. A mean score of stability index was calculated from the three trials. Between each section, they were allowed to take a break for three minutes to minimize the effects of muscles fatigue on the results. The single-leg balance ability index was measured by overall stability score, which was reported to the best indicator of the dynamic balance ability [27].

\section{Statistical analysis}

Statistical analysis was performed using SPSS version 22 (SPSS Inc., Chicago, IL, USA) and MedCalc for Windows, version 15.0 (MedCalc Software, Ostend, Belgium). Normality of all data was checked using the Shpiro-Wilk test $(p>0.05)$. To investigate the effect of pain on muscle strength, proprioception, and dynamic balance, each knee of the subjects was grouped by the intensity of pain (more painful knee versus less painful knee). Paired t-test was used to compare the difference in muscle strength, joint proprioception, and dynamic balance ability between the two groups. Pearson's correlation coefficients were used to test the correlation between the muscle strength at a different angle of velocities and the other two parameters including knee joint proprioception and dynamic balance ability. Only when the coefficient is more than 0.7 or minus than -0.7 , the relationship between variates is considered strong. A backward multiple regression analysis was performed to calculate the coefficient of factors affecting the dynamic balance. Statistical significance was accepted at a $p<0.05$.

\section{Results}

There was the total of 40 female patients in this study. The detail of the demographic data was shown in Table 1.

Effect of knee pain on knee strength, proprioception, and dynamic balance

With respect to knee extensor muscle strength, the more painful knees showed significantly lower strength than the less painful knees at angular velocities of both $60 \%$ $(54.98 \pm 13.36 \mathrm{Nm}$ vs. $63.00 \pm 9.96 \mathrm{Nm}, p=0.01)$ and $180^{\circ} / \mathrm{s}(37.78 \pm 9.55 \mathrm{Nm}$ vs $44.68 \pm 11.77 \mathrm{Nm}, p=0.01)$.
Table 1 Demographics and characteristics of study subjects $(N=40)$

\begin{tabular}{ll}
\hline Characteristics & Mean $\pm \mathrm{SD}$ \\
\hline Age (years) & $68.3 \pm 3.2$ \\
Height $(\mathrm{cm})$ & $153.0 \pm 5.0$ \\
Body mass $(\mathrm{kg})$ & $53.1 \pm 6.1$ \\
Body mass index $\left(\mathrm{kg} / \mathrm{m}^{2}\right)$ & $22.6 \pm 2.6$ \\
Dominant leg (right/left) & $33 / 7$ \\
More painful knee (right/left) & $29 / 11$ \\
Visual analogue scale & \\
$\quad$ More painful knee & $5.90 \pm 1.39$ \\
$\quad$ Less painful knee & $3.52 \pm 1.37$ \\
\hline Values were presented as mean \pm standard deviation
\end{tabular}

However, there was no statistically significant difference in knee flexor muscle strength at angular velocities of 60 $\%$ s or $180 \%$ s either (Table 2 ).

The proprioception of the knee joint was measured at $15^{\circ}, 30^{\circ}$, and $45^{\circ}$ of knee flexion using the knee joint reposition tests. We found no statistically significant difference in the proprioception measurements between the knees (Table 3). However, the dynamic balance index measurement showed a significant difference between the more and less painful knees $(3.88 \pm 1.15$ vs. $3.30 \pm 1.00, p=0.01)$.

\section{Correlation between quadriceps strength, proprioception} ability, and dynamic balance measurements

There was no significant correlation between extensor muscle strength and proprioception (all $p>0.05$, shown in Table 4). There were significant associations between dynamic balance measurements and quadriceps strength at angular velocity of $60 \%$ and $180 \%(r=-0.578$, $p<0.01 ; r=-0.439, p<0.01$, respectively. Table 4, Additional file 1: Figure S1, Additional file 2: Figure S2). Multiple regression analysis showed that dynamic balance was significantly influenced by quadriceps muscle strength, either at angular velocity of $60 \%$ or $180 \%$ (coefficient $=-$ 0.047, $p<0.01$; coefficient $=-0.032, p<0.01$, Table 5).

Table 2 Isokinetic knee extensor strength

\begin{tabular}{llll}
\hline $\begin{array}{l}\text { Isokinetic peak } \\
\text { torques }(\mathrm{Nm})\end{array}$ & More painful site & Less painful site & $p$ value \\
\hline Extensor $60 \% \mathrm{sec}$ & $54.98 \pm 13.36$ & $63.00 \pm 9.96$ & $0.01^{*}$ \\
Extensor $180 \% \mathrm{sec}$ & $37.78 \pm 9.55$ & $44.68 \pm 11.77$ & $0.01^{*}$ \\
Flexor $60 \% \mathrm{sec}$ & $27.10 \pm 6.70$ & $29.88 \pm 6.57$ & 0.06 \\
Flexor $180 \% \mathrm{sec}$ & $23.30 \pm 7.25$ & $25.50 \pm 5.61$ & 0.07 \\
\hline
\end{tabular}

Values were presented as mean \pm standard deviation ${ }^{*} p<0.05$ 
Table 3 Joint proprioception and balance index ${ }^{\mathrm{a}}$

\begin{tabular}{llll}
\hline Test & More painful site & Less painful site & $p$ value \\
\hline Joint proprioception $^{\mathrm{b}}$ & & & \\
$15^{\circ}$ knee flexion & $4.50 \pm 1.00$ & $4.36 \pm 0.89$ & 0.27 \\
$30^{\circ}$ knee flexion & $4.25 \pm 1.02$ & $4.22 \pm 10.71$ & 0.89 \\
$45^{\circ}$ knee flexion & $4.12 \pm 0.79$ & $4.03 \pm 1.06$ & 0.65 \\
Dynamic balance index & $3.88 \pm 1.15$ & $3.30 \pm 1.00$ & $0.01^{*}$ \\
\hline
\end{tabular}

${ }^{a}$ Values were presented as mean \pm standard deviation

${ }^{\mathrm{b}}$ Absolute average error in degrees

${ }^{\prime}$ The higher values meant the poor balance ability

${ }^{*} p<0.05$

\section{Discussion}

The principal findings of this study are that patients had less quadriceps muscle strength at angular velocities of both $60 \%$ and $180 \%$, poor dynamic balance stability, but similar proprioceptive acuity in their more painful knees while comparing to their less painful knees. For the association between these factors, quadriceps strength is not correlated with proprioceptive acuity but moderately correlated with dynamic balance stability. That is, OA patients had weaker quadriceps muscle strength and poor dynamic balance stability in the more painful knees.

For clinical assessment of lower limb mobility in the older persons, lots of modalities were discussed in previous studies. Ward et al. used strength (leg strength), speed of movement (leg velocity, reaction time, rapid leg coordination), range of motion (ROM) (knee flexion/knee extension/ankle ROM), asymmetry (asymmetry of leg strength and knee flexion/extension ROM measures), and trunk stability (trunk extensor endurance, kyphosis) to assess the lower limb mobility in the older persons [28]. They found that baseline weaker leg strength, trunk extensor endurance, and slower leg velocity predicting a higher likelihood of persistently poor function over two years. Baseline weaker leg strength, trunk extensor endurance, and restricted knee flexion motion also predicted a higher likelihood of a decline in function [28]. According to a recent systematic review, people with knee OA had inferior performance than healthy controls on Step Test,

Table 4 Correlation between muscle strength, joint proprioception and dynamic balance

\begin{tabular}{|c|c|c|c|c|}
\hline & \multicolumn{4}{|c|}{ Quadriceps muscle strength } \\
\hline & \multicolumn{2}{|l|}{ at $60 \%$ sec } & \multicolumn{2}{|l|}{ at $180 \% \mathrm{sec}$} \\
\hline & $\overline{\text { Correlation }(r)^{a}}$ & $p$ value & Correlation $(r)^{a}$ & $p$ value \\
\hline \multicolumn{5}{|l|}{ Joint proprioception } \\
\hline $15^{\circ}$ Knee flexion & -0.051 & 0.75 & -0.106 & 0.51 \\
\hline $30^{\circ}$ knee flexion & -0.044 & 0.78 & -0.047 & 0.77 \\
\hline $45^{\circ}$ knee flexion & 0.177 & 0.27 & 0.071 & 0.66 \\
\hline Dynamic balance & -0.578 & $<0.01^{*}$ & -0.439 & $<0.01^{*}$ \\
\hline
\end{tabular}

aPearson's correlation coefficient

${ }^{*} p<0.05$
Table 5 Correlation between muscle strength, joint proprioception, pain scale, and dynamic balance index

\begin{tabular}{|c|c|c|c|c|}
\hline Independent variables & Coefficient & Standard error & $r_{\text {partial }}$ & $p$ value \\
\hline \multicolumn{5}{|l|}{ Model 1} \\
\hline $\begin{array}{l}\text { Quadriceps strength } \\
\text { at } 60 \% \mathrm{~s}\end{array}$ & -0.047 & 0.009 & -0.506 & $<0.01^{*}$ \\
\hline $\begin{array}{l}\text { Proprioception } \\
\text { (knee flexion } 30^{\circ} \text { ) }\end{array}$ & 0.214 & 0.116 & 0.208 & 0.07 \\
\hline Pain (VAS) & 0.079 & 0.062 & 0.144 & 0.21 \\
\hline \multicolumn{5}{|l|}{ Model 1} \\
\hline $\begin{array}{l}\text { Quadriceps strength } \\
\text { at } 180^{\circ} / \mathrm{s}\end{array}$ & -0.032 & 0.011 & -0.308 & $<0.01^{*}$ \\
\hline $\begin{array}{l}\text { Proprioception } \\
\left(\text { knee flexion } 30^{\circ} \text { ) }\right.\end{array}$ & 0.162 & 0.127 & 0.145 & 0.21 \\
\hline Pain (VAS) & 0.132 & 0.069 & 0.213 & 0.06 \\
\hline
\end{tabular}

VAS, visual analogues scale ${ }^{*} p<0.05$

Single-Leg Stance Test, Functional Reach Test, Tandem Stance Test, and Community Balance and Mobility Scale [29]. In our present study, we used knee muscle strength measured in isokinetic dynamometer and dynamic balance test to evaluate the lower limb function in our subjects.

Concerning the effects of knee pain on femoral muscle strength, Messier et al. [8] reported that subjects with chronic painful knee had the significant decline in quadriceps muscle strength after a 30-month period, compared with those without knee pain. Hassan et al. [11] reported that in patients with knee osteoarthritis, there was no difference in knee joint proprioception and balance stability, but quadriceps muscle strength was increased after reducing pain by local anesthetic injection into the knee joint. Also, quadriceps muscle weakness contributes to pain and physical disability in patients with knee OA $[14,15]$, and the previous studies also showed that knee extensor strength was significantly correlated with pain, stiffness, and function in patients with knee OA [30]. A large-scale population-based cohort study also showed that quadriceps muscle strength was independently associated with knee pain regardless of the radiologic stage of knee OA [31].

In this study, we investigated the effects of pain on quadriceps muscle strength in patients with knee OA by comparing the strength between the more painful knee and the less painful knee in each subject. Results showed there was a significant difference in knee extensor muscle strength between the knees, but there was no difference in the knee flexor muscle strength. These results suggest that knee pain was associated with quadriceps strength weakness, and the cause may be reflex arthrogenous muscle inhibition of quadriceps caused by pain during the muscle strength measurement. This mechanism was thought to be due to the selective inactivation or weakening of quadriceps muscle by knee pain 
or other causes (tender point, anxiety) in patients with knee arthropathy, as found in previous studies [32, 33].

Previous studies reported conflicting results concerning the effects of knee pain on proprioception. Felson et al. [34] reported the correlation between impaired knee joint proprioception and the presence and severity of knee pain, but Hall et al. [35] reported that there was no significant difference in knee joint proprioception between the more painful knee and the less painful knee within the same patient. The finding of our study also supported Hall's study. The reasons why the results of studies on the effects of pain on proprioception were inconsistent are thought to be mainly due to the following two factors. Firstly, the Multicenter Osteoarthritis Study investigated not only subjects with knee OA but also those at high risk of developing OA including obesity, patients with a history of knee injury and patients with a history of knee surgery [36]. Overweight and knee surgery both lead to muscles weakness and the decline in proprioception ability, which may lead to an inevitable bias to the findings [37]. Secondly, the method used for evaluating knee pain in our study was different from the previous studies $[34,35]$. In previous studies, the pain intensity was evaluated by participants' subjective feeling in the knees in the previous month, which was simply classified as 'yes' or 'no'. In contrast, our study involved patients who had no knee swelling and had bilateral knee pain with a VAS difference between the knees of more than two points, to ensure more reliable results were obtained.

Our study found that there were differences in balance stability between patients' more painful knee and less painful knee. Hassan et al. [11] reported that quadriceps muscle strength was significantly improved but not proprioception and balance stability after pain relief by intraarticular anesthetic injection. That is, pain level did not influence the dynamic balance stability, which is different from the finding of our study. Balance stability is a complex process that is maintained through coordinated neuromuscular reaction by the central nervous system (CNS) $[38,39]$. This also includes sensory processing information transmitted from the vestibular organ, visual and somatic sensory organs, and involves multiple factors such as knee joint proprioception, femoral muscle strength, visual and CNS reaction. Therefore, it is difficult to consider the effects of pain in isolation. However, the quadriceps muscle is the principal dynamic stabilizer of the knee joint; thus, quadriceps muscle weakness leads to instability of the knee, which may be one of the reasons for balance instability and knee pain.

This study encountered several limitations. First, the study had a relatively small number of subjects with the cross-sectional test. Thought we enrolled 40 participants in the current study, the power was still insufficient to detect the difference in primary outcomes (post-hoc analysis, power $=0.255$ ). Second, this study comprised only females aged 65 to 75 years, which limited the generalisability of this study. Third, our study did not take into account the medication patients had. However, the effects of medications are equal to both knees, and our study only included the patients with VAS scores differences of greater than two points between each knee joint, which made our study to compare the outcomes between both knees possible. Fourth, this study selected both knee OA patients with different pain intensity in each knee (VAS $>1$ ) to test the effect of pain on knee outcomes. However, no current study was available to support or against the criteria for the difference of pain score. The future study with larger sample size, enrolling male gender will be needed to validate the findings of this study.

\section{Conclusion}

In aged women with knee OA, knee pain was associated with weak quadriceps strength and poor balance ability. Thus, the pain management strategy for the older persons with knee OA should be optimized to improve lower limb function and balance stability.

\section{Additional files}

Additional file 1: Table S1. Association between balance index and quadriceps strength at $60 \%$ s. The balance index was significantly associated with quadriceps strength at $60 \% \mathrm{~s}(p<0.01)$. (TIF $49 \mathrm{~kb}$ )

Additional file 2: Table S2. Association between balance index and quadriceps strength at $180 \%$ s. The balance index was significantly associated with quadriceps strength at $180^{\circ} / \mathrm{s}(p<0.01)$. (TIF $47 \mathrm{~kb}$ )

\section{Abbreviations}

CNS: Central nervous system; OA: Osteoarthritis; ROM: Range of motion; VAS: Visual analogue scale

\section{Funding}

No funding was obtained for this study.

Availability of data and materials

The datasets generated and analyzed during the current study are not publicly available due to the guidance of the Sungkyunkwan University School of Medicine Clinical Research Ethics Board but are available from the corresponding author on reasonable request.

\section{Authors' contributions}

DKK and PK were responsible for the study concept and design and drafting of the manuscript. DKK and PK performed the test, data acquisition, analysis, and interpretation of data. LTK provided consultation for statistics and methodology. LTK and WHP were in charge of the study concept and design, supervised the study and critically revised the manuscript for important intellectual content. All authors read and approved the final manuscript.

Ethics approval and consent to participate

This study was approved by the Sungkyunkwan University School of Medicine Clinical Research Ethics Board (SMC 2017-05-084-001). All participants were informed and agreed to join this study. The written consents were obtained from all participants. 


\section{Consent for publication}

Participants agreed and signed the consent for publication of all images and data gathering during the study.

\section{Competing interests}

The authors declare that they have no competing interests

\section{Publisher's Note}

Springer Nature remains neutral with regard to jurisdictional claims in published maps and institutional affiliations.

\section{Author details}

${ }^{1}$ Department of Sports Medicine Center, Samsung Medical Center, Sungkyunkwan University School of Medicine, 81 Irwon-ro, Gangnam-gu, Seoul 135-710, South Korea. ${ }^{2}$ Sports Medicine Center, Department of Orthopaedic Surgery, Chang Gung Memorial Hospital, No. 6 West Sec, Chia-Pu Road, Putz city, Chiayi 613 , Taiwan. ${ }^{3}$ Chang Gung University of Science and Technology, Chiayi, Taiwan.

\section{Received: 13 February 2018 Accepted: 1 October 2018} Published online: 17 October 2018

\section{References}

1. Neogi T. The epidemiology and impact of pain in osteoarthritis. Osteoarthr Cartil. 2013;21:1145-53

2. Sezer I, Illeez OG, Tuna SD, Balci N. The relationship between knee osteoarthritis and osteoporosis. Eurasian J Med. 2010;42:124-7.

3. Bindawas SM, Vennu V. Longitudinal effects of physical inactivity and obesity on gait speed in older adults with frequent knee pain: data from the osteoarthritis initiative. Int J Environ Res Public Health. 2015;12:1849-63.

4. Fransen M, McConnell S, Bell M. Therapeutic exercise for people with osteoarthritis of the hip or knee: a systematic review. J Rheumatol. 2002;29: 1737-45.

5. Palmieri-Smith RM, Thomas AC, Karvonen-Gutierrez C, Sowers MF. Isometric quadriceps strength in women with mild, moderate, and severe knee osteoarthritis. Am J Phys Med Rehabil. 2010:89:541-8.

6. Perry MC, Carville SF, Smith IC, Rutherford OM, Newham DJ. Strength, power output and symmetry of leg muscles: effect of age and history of falling. Eur J Appl Physiol. 2007;100:553-61.

7. Sharma L, Cahue S, Song J, Hayes K, Pai YC, Dunlop D. Physical functioning over three years in knee osteoarthritis: role of psychosocial, local mechanical, and neuromuscular factors. Arthritis Rheum. 2003;48:3359-70.

8. Messier SP, Glasser JL, Ettinger WH Jr, Craven TE, Miller ME. Declines in strength and balance in older adults with chronic knee pain: a 30-month longitudinal, observational study. Arthritis Rheum. 2002;47:141-8.

9. Segal NA, Glass NA, Torner J, Yang M, Felson DT, Sharma L, et al. Quadriceps weakness predicts risk for knee joint space narrowing in women in the MOST cohort. Osteoarthr Cartil. 2010;18:769-75.

10. Duman I, Taskaynatan MA, Mohur H, Tan AK. Assessment of the impact of proprioceptive exercises on balance and proprioception in patients with advanced knee osteoarthritis. Rheumatol Int. 2012;32:3793-8.

11. Hassan BS, Doherty SA, Mockett S, Doherty M. Effect of pain reduction on postural sway, proprioception, and quadriceps strength in subjects with knee osteoarthritis. Ann Rheum Dis. 2002;61:422-8.

12. Cho YR, Hong BY, Lim SH, Kim HW, Ko YJ, Im SA, et al. Effects of joint effusion on proprioception in patients with knee osteoarthritis: a singleblind, randomized controlled clinical trial. Osteoarthr Cartil. 2011;19:22-8.

13. Spencer JD, Hayes KC, Alexander IJ. Knee joint effusion and quadriceps reflex inhibition in man. Arch Phys Med Rehabil. 1984;65:171-7.

14. Pua $\mathrm{YH}$, Liang Z, Ong PH, Bryant AL, Lo NN, Clark RA. Associations of knee extensor strength and standing balance with physical function in knee osteoarthritis. Arthritis Care Res (Hoboken). 2011;63:1706-14.

15. Segal NA, Torner JC, Felson D, Niu J, Sharma L, Lewis CE, et al. Effect of thigh strength on incident radiographic and symptomatic knee osteoarthritis in a longitudinal cohort. Arthritis Rheum. 2009:61:1210-7.

16. Altman $R$, Asch $E$, Bloch D, Bole G, Borenstein D, Brandt $K$, et al. Development of criteria for the classification and reporting of osteoarthritis. Classification of osteoarthritis of the knee. Diagnostic and Therapeutic Criteria Committee of the American Rheumatism Association Arthritis Rheum. 1986;29:1039-49.
17. Kellgren JH, Lawrence JS. Radiological assessment of osteoarthrosis. Ann Rheum Dis. 1957:16:494-502.

18. Lee DH, Lee JH, Ahn SE, Park MJ. Effect of time after anterior cruciate ligament tears on proprioception and postural stability. PLoS One. 2010;10(9):e0139038.

19. von Elm E, Altman DG, Egger M, Pocock SJ, Gøtzsche PC, Vandenbroucke $J P$, et al. The strengthening the reporting of observational studies in epidemiology (STROBE) statement: guidelines for reporting observational studies. PLoS Med. 4:e296.

20. Pincivero DM, Lephart SM, Karunakara RG. Effects of rest interval on isokinetic strength and functional performance after short-term high intensity training. Br J Sports Med. 1997;31:229-34.

21. Gür H, Cakin N. Muscle mass, isokinetic torque, and functional capacity in women with osteoarthritis of the knee. Arch Phys Med Rehabil. 2003:84:1534-41.

22. Kean CO, Birmingham TB, Garland SJ, Bryant DM, Giffin JR. Minimal detectable change in quadriceps strength and voluntary muscle activation in patients with knee osteoarthritis. Arch Phys Med Rehabil. 2010;91:1447-51.

23. Hortobagyi T, Garry J, Holbert D, Devita P. Aberrations in the control of quadriceps muscle force in patients with knee osteoarthritis. Arthritis Care Res. 2004;51:562-9.

24. Warren PJ, Olanlokun TK, Cobb AG, Bentley G. Proprioception after knee arthroplasty. the influence of prosthetic design Clin Orthop Relat Res. 1993; (297):182-7.

25. Wikstrom EA, Tillman MD, Chmielewski TL, Borsa PA. Measurement and evaluation of dynamic joint stability of the knee and ankle after injury. Sports Med. 2006;36:393-410.

26. Rozzi SL, Lephart SM, Fu FH. Effects of muscular fatigue on knee joint laxity and neuromuscular characteristics of male and female athletes. J Athl Train. 1999:34:106-14

27. Testerman C, Griend RV. Evaluation of ankle instability using the Biodex stability systems. Foot Ankle Int. 1999;20:317-21.

28. Ward RE, Beauchamp MK, Latham NK, Leveille SG, Percac-Lima S, Kurlinski L, et al. Neuromuscular impairments contributing to persistently poor and declining lower-extremity mobility among older adults: new findings informing geriatric rehabilitation. Arch Phys Med Rehabil. 2016;97:1316-22. https://doi.org/10.1016/j.apmr.2016.03.003 Epub 2016 Apr 4.

29. Hatfield GL, Morrison A, Wenman M, Hammond CA, Hunt MA. Clinical tests of standing balance in the knee osteoarthritis population: systematic review and meta-analysis. Phys Ther. 2016;96:324-37. https://doi.org/10.2522/ptj. 20150025 Epub 2015 Jul 16.

30. Serrão PR, Gramani-Say K, Lessi GC, Mattiello SM. Knee extensor torque of men with early degrees of osteoarthritis is associated with pain, stiffness and function. Rev Bras Fisioter. 2012:16:289-94.

31. Muraki S, Akune T, Teraguchi M, Kagotani R, Asai Y, Yoshida N, et al. Quadriceps muscle strength, radiographic knee osteoarthritis and knee pain: the ROAD study. BMC Musculoskelet Disord. 2015;16:305. https://doi.org/10. 1186/s12891-015-0737-5.

32. Sahin N, Baskent A, Cakmak A, Salli A, Ugurlu H, Berker E. Evaluation of knee proprioception and effects of proprioception exercise in patients with benign joint hypermobility syndrome. Rheumatol Int. 2008;28:995-1000.

33. Takacs J, Carpenter MG, Garland JS, Hunt MA. The role of neuromuscular changes in aging and knee osteoarthritis on dynamic postural control. Aging Dis. 2013:4:84-99.

34. Felson DT, Gross KD, Nevitt MC, Yang M, Lane NE, Torner JC, et al. The effects of impaired joint position sense on the development and progression of pain and structural damage in knee osteoarthritis. Arthritis Rheum. 2009:61:1070-6.

35. Hall MC, Mockett SP, Doherty M. Relative impact of radiographic osteoarthritis and pain on quadriceps strength, proprioception, static postural sway and lower limb function. Ann Rheum Dis. 2006;65:865-70.

36. Felson DT, Nevitt MC. Epidemiologic studies for osteoarthritis: new versus conventional study design approaches. Rheum Dis Clin N Am. 2004;30:783-97.

37. Wang L, Li JX, Xu DQ, Hong YL. Proprioception of ankle and knee joints in obese boys and nonobese boys. Med Sci Monit. 2008;14:CR129-35.

38. Koceja DM, Allway D, Earles DR. Age differences in postural sway during volitional head movement. Arch Phys Med Rehabil. 1999:80:1537-41.

39. Whipple R, Wolfson L, Derby C, Singh D, Tobin J. Altered sensory function and balance in older persons. J Gerontol. 1993:48:71-6. 